\title{
Epidemiology of pulmonary disease due to nontuberculous mycobacteria in Southern China, 2013-2016
}

Yaoju Tan ${ }^{1 \dagger}$, Biyi Su ${ }^{1 \dagger}$, Wei Shu ${ }^{2}$, Xingshan Cai ${ }^{1}$, Shaojia Kuang ${ }^{1}$, Haobin Kuang ${ }^{1}$, Jianxiong Liu ${ }^{1 *}$ and Yu Pang ${ }^{2^{*}}$

\begin{abstract}
Background: Pulmonary nontuberculous mycobacteria (NTM) disease is of increasing public health concern in China. Information is limited regarding risk factors associated with this disease in China. The objective of this study was to describe the epidemiology of pulmonary disease due to NTM in Southern China.

Methods: We retrospectively reviewed the medical records of pulmonary NTM patients registered in the Guangzhou Chest Hospital with positive mycobacterial cultures during 2013-2016. We described sex, age, residence, treatment history, laboratory examination results and comorbidities of pulmonary NTM patients.

Results: Among the 607 NTM cases, the most prevalent species were Mycobacterium avium complex (44.5\%), Mycobacterium abscessus complex (40.5\%), Mycobacterium kansasii (10.0\%) and Mycobacterium fortuitum (2.8\%). The male:female ratio was significantly lower among patients infected with rapidly growing mycobacteria (RGM) than among those with slowly growing mycobacteria (SGM). The risk of developing SGM disease significantly increased with advancing age. In addition, pulmonary RGM diseases were more common in migrant population than resident population. Notably, patients with pulmonary RGM diseases were significantly more likely to have bronchiectasis underlying noted than those with SGM diseases. No significant difference was observed in in vitro drug susceptibility among NTM species.

Conclusion: Our data illustrate that the M. avium complex is the most predominant causative agent of pulmonary NTM disease in Southern China. Female, migrant population, the presence of bronchiectasis are independent risk factors for pulmonary diseases due to RGM. In addition, the prevalence of SGM increases significantly with advancing age.
\end{abstract}

Keywords: Nontuberculous mycobacteria, Epidemiology, Slowly growing mycobacteria, Rapidly growing mycobacteria, Comorbidity

\section{Background}

Nontuberculous mycobacteria (NTM) are a heterogeneous group of species other than the Mycobacterium tuberculosis complex and Mycobacterium leprae [1]. As the etiologic agents, NTM have been found in a variety of environmental sources, such as soil, water and

\footnotetext{
*Correspondence: Ljxer64@qq.com; pangyupound@163.com

${ }^{\dagger}$ Yaoju Tan and Biyi Su contributed equally to this work.

'Department of Clinical Laboratory, Guangzhou Chest Hospital, State Key Laboratory of Respiratory Disease, No. 62, Hengzhigang Road, Yuexiu District, Guangzhou, Guangdong Province 510095, People's Republic of China ${ }^{2}$ National Clinical Laboratory on Tuberculosis, Beijing Key laboratory on Drug-resistant Tuberculosis Research, Beijing Chest Hospital, Beijing Tuberculosis and Thoracic Tumor Institute, No. 9, Beiguan Street, Tongzhou District, Beijing 101149, People's Republic of China
}

aerosols [2]. Despite being less pathogenic than $M$. tuberculosis, these environmental bacteria are associated with a wide array of clinical diseases, especially in HIV-infected patients or those with immunodeficiencies [3]. Notably, NTM disease incidence has increased significantly during the past decade [3], while this emerging disease is given a lower public health priority as compared with tuberculosis due to lack of definitive evidence of person-to-person transmission of NTM [4]. The most available data on NTM infections come from sentinel laboratory-based surveillance studies $[5,6]$, which makes it difficult to distinguish between colonizers and causative pathogens among these positive mycobacteria

(C) The Author(s). 2018 Open Access This article is distributed under the terms of the Creative Commons Attribution 4.0 International License (http://creativecommons.org/licenses/by/4.0/), which permits unrestricted use, distribution, and 
cultures [7]; consequently, the exact distribution of mycobacteria species among patients is not well known, especially in high-TB-burden settings.

Although China has achieved impressive reductions in TB prevalence and mortality over the past 20 years [8], NTM infections have become a serious issue, accounting for about one quarter of mycobacterial patient isolates according to the national population-based data [9], triggering public health concerns. More importantly, NTM prevalence varies greatly across China, and Southern China has a significantly higher proportion of NTM infection [1]. In addition, national epidemiological data have revealed that NTM species distribution differs significantly by region, reflecting the diversity of species distribution in the local environment [3]. Given that NTM species differ significantly in pathogenicity and drug susceptibility profiles, understanding this regional diversity is a major priority for optimizing appropriate treatment regimen. Unfortunately, previous reports regarding this issue are lacking, and few laboratory data were mainly on the basis of NTM isolates from microbiology laboratory, which made it difficult to differentiate NTM diseases from host respiratory colonization $[1,10]$. Furthermore, information is limited regarding risk factors associated with this disease, thereby hampering attempts to implement effective infection control programmes. The objective of this study was to describe the prevalence of NTM species among pulmonary NTM patients in regional tuberculosis clinical centre in Southern China between 2013 and 2016. We also aimed to identify demographic and clinical factors associated with pulmonary NTM diseases between slowly growing mycobacteria (SGM) and rapidly growing mycobacteria (RGM).

\section{Methods}

\section{Study design and population}

This study was conducted at the Guangzhou Chest Hospital, an 800-bed regional tuberculosis clinical centre in Southern China. We retrospectively reviewed the medical records of pulmonary NTM patients registered in the hospital with positive mycobacterial cultures during 2013-2016. Factors that were assessed in this study included demographic and clinical characteristics, such as sex, age, residence, treatment history, laboratory examination results and comorbidities. The definition of NTM lung disease met the criteria established by the American Thoracic Society (ATS) in 2007 [11], including clinical symptoms and abnormal chest radiograph suggestive of pulmonary TB or NTM diseases; isolation of the same NTM species from more than two sputum specimens collected at different time points; and exclusion of other differential diagnoses. In addition, the residents were defined as individuals with the local household registration of Guangzhou, while the migrants were defined as individuals without local household registration of Guangzhou.

\section{Laboratory examination}

Media supplied with paranitrobenzoic acid was used for differential identification of Mycobacterium tuberculosis (MTB) complex and NTM. The NTM strains identified by conventional biochemical method were subcultured on the Löwenstein-Jensen (L-J) medium [1]. Colonies were scraped from the surface of L-J medium, and transferred to $500 \mu \mathrm{L}$ Tris-EDTA (TE) buffer. The was heated at $95{ }^{\circ} \mathrm{C}$ for $30 \mathrm{~min}$ in a water bath, and the supernatant was used as DNA template for PCR amplification. The commercial Biochip test was performed for species identification of mycobacterium according to the manufacturer's instructions [12]. In addition, the isolates identified as Mycobacterium chelonae-Mycobacterium abscessus group by Biochip were further divided into subspecies with the sequencing of multiple genes, including $16 \mathrm{~S}$ rRNA, hsp65, rpoB, and 16S-23S rRNA internal transcribed spacer (ITS) sequence as previously reported [9]. The PCR products were sent to Ruibo Company (Beijing, China) for DNA sequencing service. Nucleotide sequences were aligned with the homologous sequences of the reference mycobacteria strains by using multiple sequence alignments via the BLAST web pages (http://www.ncbi.nlm.nih.gov/BLAST).

\section{Drug susceptibility testing}

The in vitro drug susceptibility of $M$. abscessus complex was determined with a broth microdilution method based on the guidelines from the Clinical and Laboratory Standards Institute (CLSI) [13]. Eight antimicrobial agents were enrolled in this study, including amikacin, clarithromycin, linezolid, tobramycin, cefoxitin, ciprofloxacin, doxycycline and imipenem. The breakpoint values to distinguish susceptibility and resistance for drugs were followed as recommendation from CLSI [13]. For M. avium and M. intracellulare isolates, three agents were selected for MIC assessment, including clarithromycin, moxifloxacin and linezolid. The in vitro drug susceptibility for these drugs were evaluated with a broth microdilution method, and their breakpoint values were followed the recommendation from CLSI [13].

\section{Statistical analysis}

All collected data were entered using Epi Data version 3.1 (EpiData Association, Odense, Denmark). Each entry was cross checked independently to ensure the data quality. The predictor variables of age, sex, residence, previous history for tuberculosis, and comorbidity were tested for association with various NTM diseases using univariate and multivariate logistic analysis. The level of significance of univariate analysis was 0.05 , and that for 
inclusion in the multivariate model was 0.15 . Association between NTM diseases and predictor variables was calculated using adjusted odds ratio and $95 \%$ confidence interval. In addition, comparison of rate of drug resistance between different NTM species was evaluated by chi-square and Fisher's exact tests. Differences were considered to be statistically significant at $P<0.05$. We conducted analyses by using SPSS version 20.0 (SPSS Inc., Chicago, USA).

\section{Results}

\section{Proportion of different NTM species}

A total of 607 pulmonary NTM patients were enrolled during January 1, 2013-December 31, 2017. Among the 607 NTM cases, the most prevalent species were $M$. avium complex (MAC, 270 isolates, 44.5\%), M. abscessus complex (MABC, 246 isolates, 40.5\%), M. kansasii (61 isolates, $10.0 \%$ ) and $M$. fortuitum (17 isolates, 2.8\%). These four groups accounted for $97.9 \%$ of all mycobacteria identified. Of $270 \mathrm{M}$. avium complex isolates, there were $171 \mathrm{M}$. intracellulare $(63.3 \%, 171 / 270)$ and $99 \mathrm{M}$. avium $(36.7 \%, 99 / 270)$ isolates, respectively. In addition, $58.9 \%(145 / 246)$ of $M$. abscessus complex isolates were M. abscessus subspecies abscessus, and the remining 41.1\% (101/246) belonged to M. abscessus subspecies massiliense (Fig. 1).

\section{Factors associated with SGM and RGM infections}

Comparison in demographic and clinical characteristics of NTM patients between SGM and RGM is summarized in Table 1 . The male:female ratio was significantly lower among patients infected with RGM than among those with SGM [adjusted odds ratio (aOR): 0.526, 95\% confidence interval ( $95 \% \mathrm{CI}): 0.429-0.862 ; P=0.005]$. In addition, the risk of developing RGM disease significantly decreased with advancing age. Compared with patients with SGM, the adjusted odds ratios were 0.488 (95\% CI: $0.287-0.827$ ) for $40-60$ years group and 0.395 (95\% CI: $0.235-0.666$ ) for $>60$ years group, respectively. We also found that the prevalence of infection caused by RGM and SGM differed significantly in resident and migrant population, and pulmonary RGM diseases were more common in migrant population than resident population (aOR: 1.551; 95\% CI: 1.092-2.202; $P=0.014$ ). Notably, patients with pulmonary RGM diseases were significantly more likely to have bronchiectasis underlying noted than those with SGM diseases (aOR: 1.521; 95\% CI: $1.064-2.176 ; P=0.021)$. In contrast, there were no other differences regarding TB history or comorbidities noted between SGM and RGM, respectively $(P>0.05)$.

\section{In vitro drug susceptibility profiles of MAC and MABC}

We further analysed the in vitro drug susceptibility profiles of $M$. avium complex and $M$. abscessus complex. As shown in Table 2, clarithromycin was the most highly active agent against $M$. avium complex, and the percentages of resistant strains were $4.2 \%$ (4/95) for $M$. avium and 3.8\% (6/159) for $M$. intracellulare, respectively. Moxifloxacin and linezolid also showed potent activity against $M$. avium complex. There were $5(5.3 \%) M$. avium isolates and $8(5.0 \%) M$. intracellulare isolates resistant to moxifloxacin. For linezolid, the proportions of resistant isolates were $11.6 \%(11 / 95)$ for $M$. avium and $8.2 \%$ (13/159) for $M$. intracellulare, respectively. Of the antimicrobial agents tested, amikacin, clarithromycin, linezolid and tobramycin showed highly active against $M$. abscessus complex, and less than $5 \%$ of $M$.

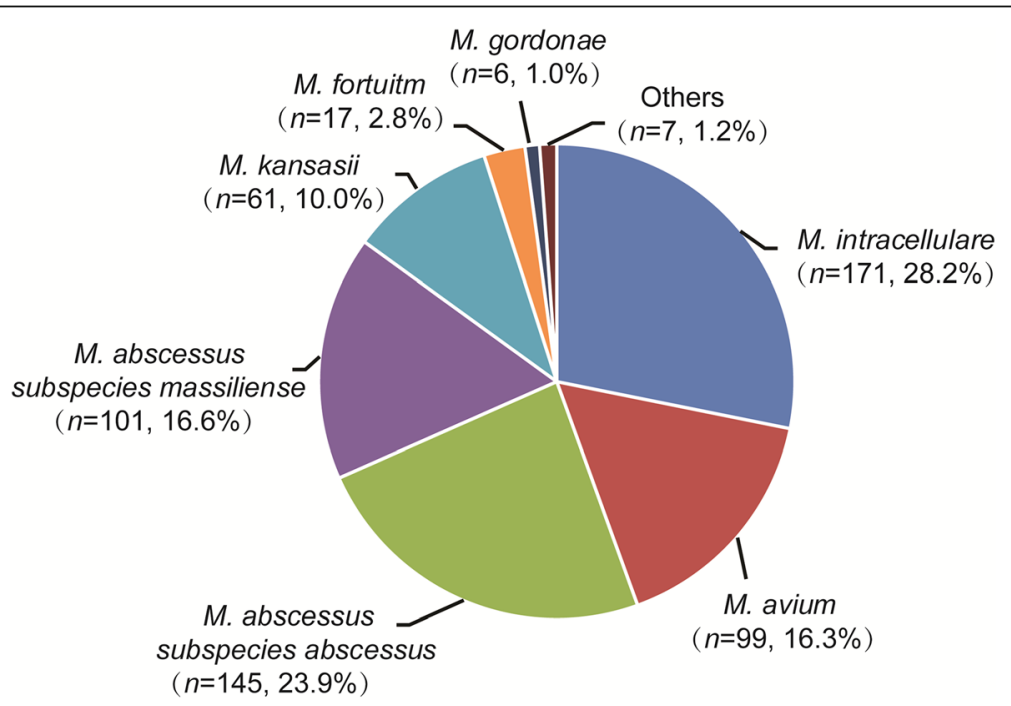

Fig. 1 Distribution of nontuberculous mycobacteria species isolated from pulmonary NTM patients in South China 
Table 1 Comparison in demographic and clinical characteristics of NTM patients between slowly growing mycobacteria and rapidly growing mycobacteria at Guangzhou Chest Hospital, China, January 1, 2013 to December 31, 2017

\begin{tabular}{|c|c|c|c|c|c|c|}
\hline \multirow[t]{2}{*}{ Characteristics } & \multicolumn{2}{|c|}{ No. of pulmonary NTM cases (\%) } & \multicolumn{2}{|l|}{ Univariate analysis } & \multicolumn{2}{|l|}{ Multivariate analysis } \\
\hline & $\begin{array}{l}\text { SGM } \\
(n=344)\end{array}$ & $\begin{array}{l}\text { RGM } \\
(n=263)\end{array}$ & OR $(95 \% \mathrm{Cl})$ & $P$ value & $\mathrm{OR}(95 \% \mathrm{Cl})$ & $P$ value \\
\hline \multicolumn{7}{|l|}{ Gender } \\
\hline Female & $170(49.4)$ & $171(65.0)$ & $0.526(0.378-0.731)$ & $<0.001$ & $0.608(0.429-0.862)$ & 0.005 \\
\hline Male & $174(50.6)$ & $92(35.0)$ & 1.000 & - & 1.000 & - \\
\hline \multicolumn{7}{|c|}{ Age group (years) } \\
\hline $18-40$ & $34(9.9)$ & $53(20.2)$ & 1.000 & - & 1.000 & - \\
\hline $40-60$ & $122(35.5)$ & $106(40.3)$ & $0.557(0.337-0.922)$ & 0.023 & $0.488(0.287-0.827)$ & 0.008 \\
\hline$>60$ & $188(54.7)$ & $104(39.5)$ & $0.355(0.217-0.581)$ & $<0.001$ & $0.395(0.235-0.666)$ & $<0.001$ \\
\hline \multicolumn{7}{|l|}{ TB history } \\
\hline No & $149(43.8)$ & $96(36.5)$ & 1.000 & - & & \\
\hline Yes & $195(57.4)$ & $167(63.5)$ & $1.329(0.953-1.852)$ & 0.093 & & \\
\hline \multicolumn{7}{|l|}{ Population } \\
\hline Residence & $168(48.8)$ & 89 (33.8) & 1.000 & - & 1.000 & - \\
\hline Migration & $176(51.2)$ & $174(66.2)$ & $1.855(1.331-2.585)$ & $<0.001$ & $1.551(1.092-2.202)$ & 0.014 \\
\hline \multicolumn{7}{|l|}{ Diabetes } \\
\hline No & $328(95.3)$ & $252(95.8)$ & 1.000 & - & & \\
\hline Yes & $16(4.7)$ & $11(4.2)$ & $0.895(0.408-1.962)$ & 0.895 & & \\
\hline \multicolumn{7}{|l|}{ Bronchiectasis } \\
\hline No & $158(45.9)$ & $93(35.4)$ & 1.000 & - & 1.000 & - \\
\hline Yes & $186(54.1)$ & $170(64.6)$ & $1.553(1.116-2.16)$ & 0.009 & $1.521(1.064-2.176)$ & 0.021 \\
\hline \multicolumn{7}{|l|}{ COPD } \\
\hline No & $326(94.8)$ & $256(97.3)$ & 1.000 & - & & \\
\hline Yes & $18(5.2)$ & $7(2.7)$ & $0.495(0.204-1.204)$ & 0.121 & & \\
\hline \multicolumn{7}{|l|}{ Tumor } \\
\hline No & $321(93.3)$ & $251(95.4)$ & 1.000 & - & & \\
\hline Yes & $23(6.7)$ & $12(4.6)$ & $0.667(0.326-1.367)$ & 0.269 & & \\
\hline
\end{tabular}

SGM slowly growing mycobacteria, $R G M$ rapidly growing mycobacteria, COPD chronic obstructive pulmonary disease, OR odds ratio, $95 \% \mathrm{Cl} 95 \%$ confidence interval

avium and $M$. intracellulare were resistant to each drug, respectively. In addition, cefoxitin had moderate activity against $M$. abscessus complex, and the percentages of cefoxitin -resistance were observed in 33.8\% (46/136) of M. abscessus subspecies abscessus and 25.3\% (24/95) of

Table 2 Comparison of in vitro drug susceptibility profiles between $M$. intracellulare and $M$. avium isolates

\begin{tabular}{llll}
\hline Antimicrobial & \multicolumn{2}{l}{ No. of resistant isolates (\%) } & $P$ value \\
\cline { 2 - 3 } & $\begin{array}{l}\text { M. avium } \\
(n=95)\end{array}$ & $\begin{array}{l}\text { M. intracellulare } \\
(n=159)\end{array}$ & \\
\hline Clarithromycin & $4(4.2)$ & $6(3.8)$ & 1.000 \\
Moxifloxacin & $5(5.3)$ & $8(5.0)$ & 1.000 \\
Linezolid & $11(11.6)$ & $13(8.2)$ & 0.370 \\
\hline
\end{tabular}

The breakpoints to establish susceptibility and resistance for clarithromycin, moxifloxacin and linezolid were followed as recommendation from Clinical and Laboratory Standards Institute (CLSI-M24-A2)
M. abscessus subspecies massiliense isolates. Statistical analysis revealed that there were no significant differences in the drug resistant rate between $M$. abscessus subspecies abscessus and M. abscessus subspecies massiliense $(P>0.05)$ (Table 3$)$.

\section{Discussion}

Pulmonary NTM disease is of increasing public health concern worldwide [6]. This study firstly describes the demographic and clinical characteristics of patients with pulmonary NTM disease in Southern China. Our study has demonstrated that the most common NTM that causes pulmonary disease in Southern China is $M$. avium complex, accounting for $44.5 \%$ of pulmonary NTM disease burden in this study, which is consistent with its predominance in other parts of the world, including the United States (85\%) [14], Denmark (81\%) 
Table 3 Comparison of in vitro drug susceptibility profiles between M. abscessus subspecies abscessus and M. abscessus subspecies massiliense isolates

\begin{tabular}{|c|c|c|c|}
\hline \multirow{2}{*}{$\begin{array}{l}\text { Antimicrobial } \\
\text { agents }\end{array}$} & \multicolumn{2}{|c|}{ No. of resistant isolates (\%) } & \multirow[t]{2}{*}{$P$ value } \\
\hline & $\begin{array}{l}\text { M. abscessus subspecies } \\
\text { abscessus }(n=136)\end{array}$ & $\begin{array}{l}\text { M. abscessus } \\
\text { subspecies massiliense } \\
(n=95)\end{array}$ & \\
\hline Amikacin & $3(2.2)$ & $2(2.1)$ & 1.000 \\
\hline Clarithromycin & $6(4.4)$ & $3(3.2)$ & 0.740 \\
\hline Linezolid & $6(4.4)$ & $2(2.1)$ & 0.476 \\
\hline Tobramycin & $6(4.4)$ & $2(2.1)$ & 0.476 \\
\hline Cefoxitin & $46(33.8)$ & $24(25.3)$ & 0.164 \\
\hline Ciprofloxacin & $86(63.2)$ & $68(71.6)$ & 0.186 \\
\hline Doxycycline & $127(93.4)$ & $94(98.9)$ & 0.050 \\
\hline Imipenem & $134(98.5)$ & $95(100.0)$ & 1.000 \\
\hline
\end{tabular}

The breakpoints to establish susceptibility and resistance for drugs were followed as recommendation from Clinical and Laboratory Standards Institute (CLSI-M24-A2)

[15] and South Korea (48\%) [16]. The second most frequently identified NTM specie is $M$. abscessus complex. Despite occurring less frequently in Northern America and Europe $[14,15]$, this species was found to generally cause $>30 \%$ of pulmonary NTM infections in India [17], Taiwan [18] and South Korea [16]. The marked geographic variation in mycobacteria species could reflect the diversity of species composition of NTM in environmental niches [3]. Interestingly, all the regions with high isolation frequency of $M$. abscessus are in Asia; we thus speculate that Asian persons may also be more susceptible to $M$. abscessus infection. In line with our hypothesis, Adjemian and colleagues found that Asian persons have an increased risk for infection with $M$. abscessus than other ethnics [19]. Hence, the ethnic factors contributing to susceptibility to different NTM species may also play an important role in the diverse geographic NTM patterns across world regions.

Another interesting finding of this study is that the frequency of NTM species from pulmonary patients differs significantly from the observations from a recent laboratory-based study in Guangzhou [1]. First, $M$. avium complex exceeds $M$. abscessus complex as the predominant causative agent of pulmonary NTM disease, which reflects the inherent difference in NTM prevalence between pulmonary diseases and colonization. The species difference of pulmonary NTM colonization may partly determine the frequency and manifestations of pulmonary NTM disease [3]. However, the variation of pathogenicity among distinct NTM species could greatly contribute to the prevalence of diseases due to NTM species [20]. A population-based study of patients with respiratory NTM isolates from the Netherlands revealed that differences in clinical relevance exist among NTM species [20]. Similar results were noted in a systematic review in Eastern Asian that M. avium complex was clinically more relevant than $M$. abscessus complex among patients meeting the ATS diagnostic criteria [21]. Although experimental evidences are limited, there is no doubt that the relative greater pathogenicity of $M$. avium complex compared with $M$. abscessus complex would increase the risk from NTM colonization to active disease in respiratory tract.

Second, M. gordonae was the third frequently isolates species in South China on the basis of previous data, accounting for $22.5 \%$ of NTM isolates [1]. In contrast, only $1 \%$ of pulmonary NTM diseases was caused by $M$. gordonae in the present study. This finding confirms that this species is a rarely isolated weak pathogen, majorly contributing to patient colonization and culture contamination rather than patient disease [3].

Third, several rare geographically restricted NTM species identified by previous study was not associated with NTM diseases [1]. Although for these species the small number of isolates decrease the reliability of conclusions, the disappearance of previous laboratory isolation of these rare species among NTM patients may reflect NTM colonization due to their weak pathogenicity for human individuals. Therefore, the significant change in the prevalence of NTM species between pulmonary diseases and colonization indicates that the pathogenicity differs by species, thereby leading to the difference in clinical relevance of the various NTM species, which should be taken into consideration in formulating the diagnostic criteria for pulmonary NTM diseases.

Pulmonary NTM disease is not uncommon, particularly among elderly females [22]. Our results demonstrate that female is more likely to be associated with the acquisition of NTM diseases caused by RGM than SGM. In agree with our observation, an early study from the United States suggested that $M$. avium complex lung disease was more common among males than females 
[23]. The gender difference in the NTM diseases may reflect differences in the immune responses during infection between RGM and SGM [24]. Generally, females exhibit greater cell-mediated immune responses to infection and vaccination than males [25], which is also important in host defense against mycobacteria. We therefore hypothesize that this instinct immunological difference may affect in SGM infections more than RGM infections.

Numerous studies have documented that pulmonary NTM infection more frequently affect elderly patients $[6,25]$. In this study, we found that the prevalence of SGM increased significantly with advancing age. Furthermore, half of SGM diseases occurred in patients $>60$ years of age. On one hand, the immunity in elderly persons is less able to produce an effective immune response after challenges with mycobacteria than the young [26]. This condition would result in greater incidence or reactivation of mycobacteria. On the other hand, the high incidence of co-morbidities presumed to affect the immune response in this population, such as diabetes, kidney failure, and immunosuppressive therapy, may favor the progression of pulmonary NTM infection. More studies are need to evaluate the relative contribution of each factor to the increased risk of pulmonary SGM diseases.

The association between bronchiectasis and NTM disease has been described by several reports [27-29]. We found that the presence of bronchiectasis appears to be more closely associated with RGM than SGM. It has been hypothesized that the impaired secretion clearance due to bronchiectasis enables NTM airway colonization and increases the risk of infection [29]. A recent report by Williams et al. compared the biofilm formation between RGM and SGM, demonstrating that M. avium complex is better equipped to grow in low-nutrient conditions than RGM by the development of more culturable biofilm [30]. Although the exact reason remains unknown, the reduced capability in the synthesis of biofilm of RGM allows these species prefer to inhabit in architectural-defected airway rather than normal airway, offering an explanation for the greater occurrence of bronchiectasis among pulmonary RGM cases.

This study is subject to several limitations. First, HIV infection has been regarded as an independent risk factor for NTM infections [31], while the HIV-positive patients were not included in this study because the HIV-positive patients with were transferred to another hospital receiving antiviral treatment. Given that M. avium infections are frequently encountered in AIDS patients [31], we may underestimate the prevalence of $M$. avium isolates in Southern China. Second, the clinical outcomes of pulmonary NTM patients were not collected in this study, because patients are not under follow-up for NTM diseases in China. As a consequence, we only analysed in vitro antibiotic susceptibility of NTM isolates rather than its correlation with treatment results. Therefore, further study is urgently needed to investigate the correlation between in vitro drug susceptibility and clinical outcomes among NTM patients. Third, another important explanation for poor response to macrolide-based chemotherapy for $M$. abscessus infections is the inducible macrolide resistance phenotype. Unfortunately, the routine detection of drug susceptibility for RGM only incubates 96-well microtiter plates for 3 days, whereas the detection of inducible resistance requires an extended incubation of plates with reading after 14 days of incubation. As a consequence, the resistance to clarithromycin for $M$. abscessus would be underestimated. Fourth, although cavitary is another major category regarding NTM pulmonary diseases, the radiological characteristics were not collected in this study due to the limited information in the medical records of patients. Nevertheless, this study provides important hints to help clinicians interpret laboratory results and recognize the risk factors associated with various NTM species.

\section{Conclusion}

In conclusion, our data illustrate that the M. avium complex is the most predominate causative agent of pulmonary NTM disease in Southern China. Female, migrant population, the presence of bronchiectasis are independent risk factors for pulmonary diseases due to RGM. In addition, the prevalence of SGM increases significantly with advancing age. In view of the growing public health concern, further studies will be carried out to determine the association between in vitro susceptibility and treatment outcome among these NTM patients, which is essential to help clinicians select effective regimens for the treatment of NTM infections.

\section{Acknowledgments \\ We thank all staffs from Guangzhou Chest Hospital for their help in carrying out this study.}

\section{Funding}

This research was supported by the Guangzhou Collaborative Innovation Major Project on Health and Medicine (201604020019), National Key Project (2015ZX10003003) and the Beijing Municipal Administration of Hospitals' Youth Programme (QML20171601).

\section{Authors' contributions}

YT, BS, WS, XC, SK, HK, JL, and YP: Substantial contributions to conception and design of, or acquisition of data or analysis and interpretation of data. $Y T, B S, J L$, and YP: Drafting the article or revising it critically for important intellectual content. All authors read and approved the final manuscript.

\section{Ethics approval and consent to participate}

This study was approved by the Ethical Committee of the Guangzhou Chest Hospital. Informed consent was waived by the institutional review board because all of the patients enrolled in this study had previously provided authorization for use of their medical records for research.

Consent for publication

Not applicable. 


\section{Competing interests}

The authors declare that they have no competing interests.

\section{Publisher's Note}

Springer Nature remains neutral with regard to jurisdictional claims in published maps and institutional affiliations.

\section{Received: 19 August 2018 Accepted: 18 October 2018}

Published online: 09 November 2018

\section{References}

1. Pang Y, Tan Y, Chen J, Li Y, Zheng H, Song Y, Zhao Y. Diversity of nontuberculous mycobacteria in eastern and southern China: a crosssectional study. Eur Respir J. 2017;49(3):1601429.

2. Falkinham JO III. Epidemiology of infection by nontuberculous mycobacteria. Clin Microbiol Rev. 1996;9(2):177-215.

3. Hoefsloot W, van Ingen J, Andrejak C, Angeby K, Bauriaud R, Bemer P, Beylis N, Boeree MJ, Cacho J, Chihota V, et al. The geographic diversity of nontuberculous mycobacteria isolated from pulmonary samples: an NTMNET collaborative study. Eur Respir J. 2013:42(6):1604-13.

4. Bryant JM, Grogono DM, Greaves D, Foweraker J, Roddick I, Inns T, Reacher M, Haworth CS, Curran MD, Harris SR, et al. Whole-genome sequencing to identify transmission of Mycobacterium abscessus between patients with cystic fibrosis: a retrospective cohort study. Lancet. 2013;381(9877):1551-60

5. Grubek-Jaworska H, Walkiewicz R, Safianowska A, Nowacka-Mazurek M, Krenke R, Przybylowski T, Chazan R. Nontuberculous mycobacterial infections among patients suspected of pulmonary tuberculosis. Eur J Clin Microbiol Infect Dis. 2009:28(7):739-44

6. McShane PJ, Glassroth J. Pulmonary disease due to nontuberculous mycobacteria: current state and new insights. Chest. 2015;148(6):1517-27.

7. Hernandez-Garduno E, Elwood RK. Increasing incidence of nontuberculous mycobacteria, Taiwan, 2000-2008. Emerg Infect Dis. 2010;16(6):1047 author reply $1047-1048$

8. Wang L, Zhang H, Ruan Y, Chin DP, Xia Y, Cheng S, Chen M, Zhao Y, Jiang S, Du X, et al. Tuberculosis prevalence in China, 1990-2010; a longitudinal analysis of national survey data. Lancet. 2014;383(9934):2057-64.

9. Zhang Z, Pang Y, Wang Y, Cohen C, Zhao Y, Liu C. Differences in risk factors and drug susceptibility between Mycobacterium avium and Mycobacterium intracellulare lung diseases in China. Int J Antimicrob Agents. 2015;45(5):491-5.

10. Shao Y, Chen C, Song H, Li G, Liu Q, Li Y, Zhu L, Martinez L, Lu W. the epidemiology and geographic distribution of nontuberculous mycobacteria clinical isolates from sputum samples in the eastern region of China. PLoS Negl Trop Dis. 2015;9(3):e0003623.

11. Griffith DE, Aksamit T, Brown-Elliott BA, Catanzaro A, Daley C, Gordin F, Holland SM, Horsburgh R, Huitt G, lademarco MF, et al. An official ATS/IDSA statement: diagnosis, treatment, and prevention of nontuberculous mycobacterial diseases. Am J Respir Crit Care Med. 2007;175(4):367-416.

12. Zhu L, Jiang G, Wang S, Wang C, Li Q, Yu H, Zhou Y, Zhao B, Huang H, Xing $W$, et al. Biochip system for rapid and accurate identification of mycobacterial species from isolates and sputum. J Clin Microbiol. 2010; 48(10):3654-60.

13. Clinical and Laboratory Standards Institute. Susceptibility testing of mycobacteria, nocardia, and other aerobic actinomycetes; approved standard, 2nd ed; CLSI document M24-A2. Clinical and Laboratory Standards Institute, Wayne. (2011).

14. Cassidy PM, Hedberg K, Saulson A, McNelly E, Winthrop KL. Nontuberculous mycobacterial disease prevalence and risk factors: a changing epidemiology. Clin Infect Dis. 2009;49(12):e124-9.

15. Andrejak C, Thomsen VO, Johansen IS, Riis A, Benfield TL, Duhaut P, Sorensen HT, Lescure FX, Thomsen RW. Nontuberculous pulmonary mycobacteriosis in Denmark: incidence and prognostic factors. Am J Respir Crit Care Med. 2010:181(5):514-21.

16. Koh WJ, Kwon OJ, Jeon K, Kim TS, Lee KS, Park YK, Bai GH. Clinical significance of nontuberculous mycobacteria isolated from respiratory specimens in Korea. Chest. 2006;129(2):341-8.

17. Prevots DR, Marras TK. Epidemiology of human pulmonary infection with nontuberculous mycobacteria: a review. Clin Chest Med. 2015;36(1):13-34.
18. Chien JY, Lai CC, Sheng WH, Yu CJ, Hsueh PR. Pulmonary infection and colonization with nontuberculous mycobacteria, Taiwan, 2000-2012. Emerg Infect Dis. 2014;20(8):1382-5.

19. Adjemian J, Frankland TB, Daida YG, Honda JR, Olivier KN, Zelazny A, Honda S, Prevots DR. Epidemiology of nontuberculous mycobacterial lung disease and tuberculosis, Hawaii. USA Emerg Infect Dis. 2017;23(3):439-47.

20. van Ingen J, Bendien SA, de Lange WC, Hoefsloot W, Dekhuijzen PN, Boeree MJ, van Soolingen D. Clinical relevance of non-tuberculous mycobacteria isolated in the Nijmegen-Arnhem region. The Netherlands Thorax. 2009;64(6):502-6.

21. Simons S, van Ingen J, Hsueh PR, Van Hung N, Dekhuijzen PN, Boeree MJ, van Soolingen D. Nontuberculous mycobacteria in respiratory tract infections. eastern Asia Emerg Infect Dis. 2011;17(3):343-9.

22. Winthrop KL, McNelley E, Kendall B, Marshall-Olson A, Morris C, Cassidy M, Saulson A, Hedberg K. Pulmonary nontuberculous mycobacterial disease prevalence and clinical features: an emerging public health disease. Am J Respir Crit Care Med. 2010;182(7):977-82.

23. O'Brien RJ, Geiter LJ, Snider DE Jr. The epidemiology of nontuberculous mycobacterial diseases in the United States. Results from a national survey Am Rev Respir Dis. 1987;135(5):1007-14.

24. vom Steeg LG, Klein SL. SeXX matters in infectious disease pathogenesis. PLoS Pathog. 2016:12(2):e1005374.

25. Klein SL, Jedlicka A, Pekosz A. The Xs and $Y$ of immune responses to viral vaccines. Lancet Infect Dis. 2010;10(5):338-49.

26. Solana R, Pawelec G, Tarazona R. Aging and innate immunity. Immunity. 2006:24(5):491-4.

27. Fowler SJ, French J, Screaton NJ, Foweraker J, Condliffe A, Haworth CS, Exley AR, Bilton D. Nontuberculous mycobacteria in bronchiectasis: prevalence and patient characteristics. Eur Respir J. 2006;28(6):1204-10.

28. Reich JM. Genesis of the nodular bronchiectasis phenotype of pulmonary disease due to nontuberculous mycobacteria. Chest. 2016;149(4):1113.

29. Cook JL. Nontuberculous mycobacteria: opportunistic environmental pathogens for predisposed hosts. Br Med Bull. 2010:96:45-59.

30. Williams MM, Yakrus MA, Arduino MJ, Cooksey RC, Crane CB, Banerjee SN, Hilborn ED, Donlan RM. Structural analysis of biofilm formation by rapidly and slowly growing nontuberculous mycobacteria. Appl Environ Microbiol. 2009:75(7):2091-8.

31. Guthertz LS, Damsker B, Bottone EJ, Ford EG, Midura TF, Janda JM. Mycobacterium avium and Mycobacterium intracellulare infections in patients with and without AIDS. J Infect Dis. 1989;160(6):1037-41.

\section{Ready to submit your research? Choose BMC and benefit from:}

- fast, convenient online submission

- thorough peer review by experienced researchers in your field

- rapid publication on acceptance

- support for research data, including large and complex data types

- gold Open Access which fosters wider collaboration and increased citations

- maximum visibility for your research: over $100 \mathrm{M}$ website views per year

At BMC, research is always in progress.

Learn more biomedcentral.com/submissions 